\title{
On the Characterization of Plane Bus Graphs
}

\author{
Till Bruckdorfer ${ }^{1}$, Stefan Felsner ${ }^{2}$, and Michael Kaufmann ${ }^{1}$ \\ 1 Wilhelm-Schickard-Institut für Informatik, Universität Tübingen, Germany, \\ \{bruckdor,mk\}anformatik.uni-tuebingen.de \\ 2 Institut für Mathematik, Technische Universität Berlin, Germany, \\ felsner@math.tu-berlin.de
}

\begin{abstract}
Bus graphs are being used for the visualization of hyperedges, for example in VLSI design. Formally, they are specified by bipartite graphs $G=$ $(B \cup V, E)$ of bus vertices $B$ realized by single horizontal and vertical segments, and point vertices $V$ that are connected orthogonally to the bus segments. The decision whether a bipartite graph admits a bus realization is NP-complete. In this paper we show that in contrast the question whether a plane bipartite graph admits a planar bus realization can be answered in polynomial time.

We first identify three necessary conditions on the partition $B=B_{V} \cup B_{H}$ of the bus vertices, here $B_{V}$ denotes the vertical and $B_{H}$ the horizontal buses. We provide a test whether good partition, i.e., a partition obeying these conditions, exist. The test is based on the computation of maximum matching on some auxiliary graph. Given a good partition we can construct a non-crossing realization of the bus graph on an $O(n) \times O(n)$ grid in linear time.
\end{abstract}

\section{Introduction}

A classical topic in the area of graph visualization is orthogonal graph drawing; related surveys can be found in $[3,11,18]$. In this drawing model each edge consists of a series of subsequent horizontal or vertical line segments. Applications can be found in e.g. VLSI design, cf. [17, 12]. In this application it may also be necessary to model hypergraphs. For example power buses on VLSI chips are often modeled as hyperedges, as well as LANs in computer network visualization. Bus graphs - as being defined later and their generalizations are a possible approach to model hyperedges. A bus-style representation might also be used when facing the visualization of highly interconnected parts of a given graph. So, cliques can be represented in a compact and comprehensive way using a bus-style model.
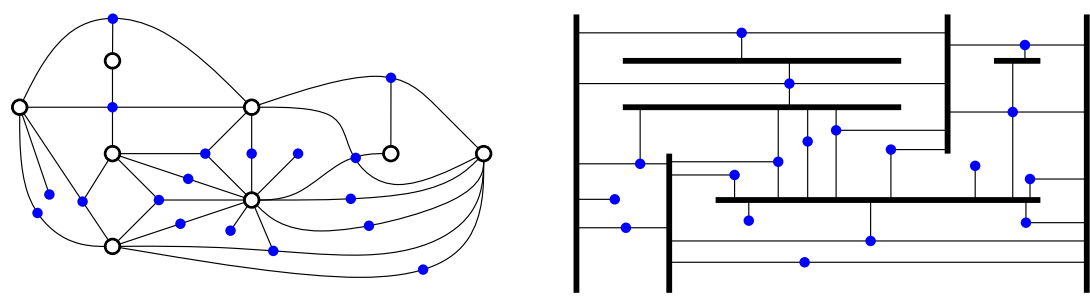

Fig. 1: An example of a plane bus graph with a planar realization. 
The bus graph approach is very much related to the classical topic of rectilinear Steiner trees, where trees are being used to connect subsets of the vertices $[8,10]$. Related are also works on rectangular drawings, rectangular duals and visibility graphs. The latter graphs are highly related to bus graphs, because connections in bus graphs enforce visibility constraints in realizations. Corresponding key concepts and surveys can be found in $[9,15,16]$ and in chapter 6 of [14]. We will use some of the methods that have been developed in this area.

We are considering the bus graph model introduced by Ada et al. [1] A bus graph is a bipartite graph $G=(B \cup V, E)$, where $E \subseteq V \times B$ and $\operatorname{deg}(v) \leq 4$ for all $v \in V$. We call vertices in $B$ bus vertices and vertices in $V$ connector vertices. A plane bus graph is a planar bus graph together with a planar embedding. A realization of a bus graph is a drawing $D$, where bus vertices are represented as horizontal or vertical line segments (bus segments), connector vertices are drawn as points, and the edges are horizontal or vertical segments (connections), i.e. segments connecting perpendicular a point with a bus segment. To distinguish between bus vertices and edges in a realization, the bus segments are drawn with bold lines, see Figure 1. A planar realization is a realization without crossings. We always assume to have a connected bus graph, since components can be considered separately.

In [1] a relation of bus graphs to hypergraphs is mentioned: the bipartite adjacency matrix of a bipartite graph can be read as incidence matrix of a hypergraph and vice versa, if vertices are contained in at most four hyperedges. Ada et al. [1] considered the problem to decide, if a bus graph has a realization and showed the NP-completeness. In this paper we consider the problem to decide if a plane bus graph has a planar realization. We show that this question in contrast to the previous result can be decided in polynomial time.

The bus segments will be drawn either vertically or horizontally. So we assign a labeling to the bus vertices that determines if they will be realized either vertically or horizontally. This labeling ensures a planar realization, if it obeys some properties. The paper is structured as follows: In Section 2 we provide necessary properties for the labeling. After that in Section 3 we give a polynomial time algorithm that tests whether a given maximal plane bus graph admits a labeling with these properties, which we call a good partition. If it exists, the algorithm also returns a good partition. In Section 4 we modify the approach so that it also works in cases where the bus graph is not maximal. In Section 5 we show how to actually produce a realization of a plane bus graph with a good partition. The approach is based on techniques from [2] and [6].

\section{Preliminaries}

In this paper we consider plane bus graphs $G=(V \cup B, E)$, i.e., planar bus graphs together with a fixed planar embedding. A diamond in a plane bus graph is a cycle $z=\left(b, v, b^{\prime}, v^{\prime}\right)$ of length four with bus vertices $b, b^{\prime}$ and connector vertices $v, v^{\prime}$ such that both $v, v^{\prime}$ have a third bus neighbour in the bounded region defined by $z$. A planar realization of a plane bus graph $G=(V \cup B, E)$, induces labels $H$ or $V$ for the bus vertices where $H$ indicates that a bus is represented by a horizontal segment in the realization, while $V$ indicates that the bus is represented by a vertical segment. Let $\Pi=\left(B_{V}, B_{H}\right)$ denote the partition of $B$ according to the label of the bus vertices. 
We observe three properties of a partition $\Pi=\left(B_{V}, B_{H}\right)$ corresponding to a planar realization:

(P1) Every connector vertex of degree $\geq 3$ has neighbors in both classes.

(P2) A connector vertex $v$ of degree 4 has cyclically alternating neighbors in both classes.

(P3) A diamond has both bus vertices $b, b^{\prime}$ in the same partition class.

The first two properties are obvious from the nature of a realization. The third property is shown in the following lemma.

Lemma 1. Let $G$ be a plane bus graph that has a realization inducing the partition $\left(B_{V}, B_{H}\right)$ of the set of buses $B$. For any diamond $z=\left(b, v, b^{\prime}, v^{\prime}\right)$ the two bus vertices $b$ and $b^{\prime}$ belong to the same class.

Proof. Suppose by contradiction that $b \in B_{H}$ and $b^{\prime} \in B_{V}$. The interior of $z$ in the planar bus realization is a polygon with six corners. Four of the corners are at contacts of connector edges and buses and two corners are at the connector vertices. Some of these corners may degenerate so that the polygon actually only has four corners. We account for four corners of size $\pi / 2$ each, where the edges meet the buses. The other two corners are at $v$ and $v^{\prime}$. Since $b$ is horizontal and $b^{\prime}$ vertical the angles at $v$ and $v^{\prime}$ have to be either $\pi / 2$ or $3 \pi / 2$. Because $v$ and $v^{\prime}$ have an additional bus neighbor in the interior the angle at each of $v$ and $v^{\prime}$ is at least $\pi$. Hence, both these angles are of size $3 \pi / 2$. The sum of interior angles is at least $4 \cdot \pi / 2+2 \cdot 3 \pi / 2=5 \pi$. A six-gon, however, has a sum of angles of $4 \pi$. The contradiction shows that $b$ and $b^{\prime}$ belong to the same class of the partition $B=B_{V} \cup B_{H}$.

Note that the outer cycle of $G$ is a diamond when the outer face of $G$ has cardinality 4 .

A partition $\Pi=\left(B_{V}, B_{H}\right)$ of the buses of a plane bus graph $G$ is called a good partition if it obeys properties (P1), (P2), and (P3).

Let $\Delta=\Delta(G)$ denote the degree of a plane bus graph $G$ which is defined as the maximum degree among the connector vertices of $G$. In the next section we consider maximal plane bus graphs and test efficiently, if they admit a good partition. The test is constructive, i.e., if the answer is yes, then a good partition is constructed.

\section{Maximal Plane Bus Graphs}

A maximal plane bus graph $G$ is a plane bipartite bus graph, where all its faces have cardinality 4 . Let $G=(V \cup B, E)$ be a maximal plane bus graph. We assume, that $G$ has no connector vertices of degree 1 or 2 , since they have no influence on the existence of a good partition.

In this section we first assume $\Delta=3$ for $G$ and give an algorithm to test $G$ if it admits a good partition and if so the algorithm returns a good partition. After that we allow $\Delta=4$ and reduce this case with a simple modification to the case $\Delta=3$.

Let $G=(V \cup B, E)$ be a plane maximal bus graph with $\Delta=3$. The connector graph $C_{G}=\left(V_{C}, E_{C}\right)$ of $G$ consists of all the connector vertices $V_{C}=V$ and edges 
$\left(v, v^{\prime}\right) \in E_{C}$, if $v$ and $v^{\prime}$ are both incident to the same face of the plane embedding of $G$. The connector graph is helpful because it allows the translation of the problem of finding a good partition for $G$ to the problem of finding an appropriate perfect matching in $C_{G}$, summarized in Proposition 1 and Proposition 2 and illustrated in Figure 2.
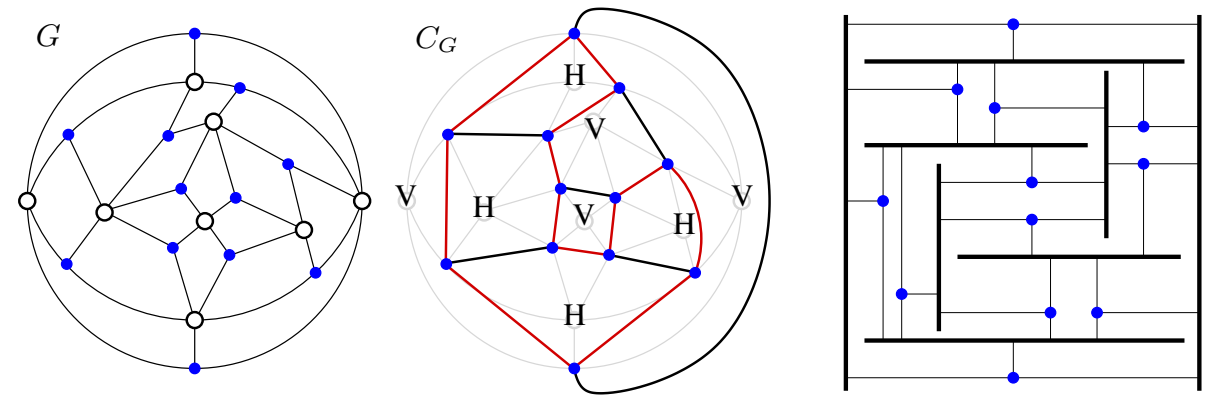

Fig. 2: A maximal plane bus graph, the connector graph with a matching and its good partition, and a corresponding bus representation.

The first property (P1) of a good partition $\Pi$ of $G$ requires that every connector vertex $v$ has two adjacent bus vertices in one partition class and one in the other. If $b$ and $b^{\prime}$ are neighbors of $v$ in $G$ with the same label, then there is a connector vertex $v^{\prime}$ sharing a face with $v, b$, and $b^{\prime}$, since every face has cardinality 4 . When looking at $v^{\prime}$ the two neighbors $b$ and $b^{\prime}$ are again the two in a common partition class. Hence, property (P1) of a good partition of $G$ induces a perfect matching on $C_{G}$.

Conversely a perfect matching $M$ of $C_{G}$ induces a labeling of the bus vertices. Removing the matching edges from $C_{G}$ leaves a 2-regular graph, i.e., a disjoint collection of cycles. The regions defined by this collection of cycles can be 2-colored with colors $V$ and $H$ such that each cycle has regions of different colors on its sides. Let $B_{V}$ be the set of bus vertices in faces colored with $V$, and $B_{H}$ be the set of bus vertices in faces colored with $H$. This yields a partition satisfying (P1) because every connector vertex is on a cycle and has a bus neighbour in each of the two faces bounded by the cycle.

Since $\Delta=3$, the second property (P2) is void.

Consider a diamond $z=\left(b, v, b^{\prime}, v^{\prime}\right)$ in $G$. Each of $v, v^{\prime}$, has exactly one edge $e, e^{\prime}$, in $C_{G}$, that corresponds to a face of the outside of $z$. Since (P3) forces equal labels for $b, b^{\prime}$, the faces represented by $e, e^{\prime}$ have equally labeled incident bus vertices $\left(b, b^{\prime}\right)$ and thus $e, e^{\prime}$ must be in a matching of $C_{G}$. We define the set $E_{d}$ of edges forced by diamonds as the set of edges consisting of the two outside edges $e, e^{\prime}$ in $C_{G}$ for each diamond $z$ of $G$. We have thus shown that a perfect matching $M$ induced by a good partition contains $E_{d}$.

Conversely, if $E_{d}$ is contained in a perfect matching $M$ of $C_{G}$, then the bus vertices $b, b^{\prime}$ of each diamond are in the same partition class and thus $G$ has a partition, that satisfies property (P3). The findings are summarized in the following proposition. 
Proposition 1. Let $G$ be a maximal plane bus graph with $\Delta=3$ and $C_{G}$ its connector graph and $E_{d}$ the set of edges of $C_{G}$ forced by diamonds. Then $G$ admits a good partition, iff $C_{G}$ has a perfect matching $M$, with $E_{d} \subseteq M$.

Now we allow $\Delta=4$ for a maximal plane bus graph $G$. To transform $G$ into a plane bus graph $G^{\prime}$ with $\Delta=3$, we split every connector vertex $v$ of degree 4 into two connector vertices $v^{\prime}, v^{\prime \prime}$, both of degree 3 in the following way: let $b_{1}, b_{2}, b_{3}, b_{4}$ be the adjacent bus vertices of $v$ in consecutive cyclic order around $v$. Remove $v$ and its incident edges and connect the new introduced vertices $v^{\prime}, v^{\prime \prime}$ with edges $\left(b_{1}, v^{\prime}\right)$, $\left(b_{2}, v^{\prime}\right),\left(b_{3}, v^{\prime}\right),\left(b_{3}, v^{\prime \prime}\right),\left(b_{4}, v^{\prime \prime}\right),\left(b_{1}, v^{\prime \prime}\right)$. The connector graph $C_{G}$ is obtained from $C_{G^{\prime}}$ by contracting the edges $\left(v^{\prime}, v^{\prime \prime}\right)$ corresponding to the pairs $v^{\prime}, v^{\prime \prime}$ that have been obtained by splitting a vertex of degree 4 . Define the set $E_{s}$ of edges forced by splits of $C_{G^{\prime}}$ as the set of these edges $\left(v^{\prime}, v^{\prime \prime}\right)$.

If $G$ has a partition satisfying property (P2), then we have to ensure alternating labels for the neighbours of $v$ in $G$. This forces $\left(v^{\prime}, v^{\prime \prime}\right) \in E_{s}$ to be a matching edge in $C_{G^{\prime}}$. Conversely if $\left(v^{\prime}, v^{\prime \prime}\right) \in E_{s}$ is a matching edge, then the common neighbours $b_{1}, b_{3}$ have the same label. Since $v^{\prime}, v^{\prime \prime}$ have both degree 3 and two of their neighbours have equal label, the third neighbour (for each of $v^{\prime}, v^{\prime \prime}$ ) has a different label, i.e. $b_{2}$ and $b_{4}$ have both different label compared to the label of $b_{1}, b_{3}$, hence, $v$ obeys property (P2). So in total a partition $\Pi$ of $G$ satisfies property (P2), iff the edges $E_{s}$ are contained in a matching of $C_{G^{\prime}}$. For notational simplicity we denote the connector graph $C_{G^{\prime}}$ of the transformed graph $G^{\prime}$ by $C_{G}$. An example for a maximal plane bus graph with its connector graph showing the edges of $E_{d} \cup E_{s}$ is shown in Figure 3.

Proposition 2. Let $G$ be a maximal plane bus graph with $\Delta=4$ and $C_{G}$ its connector graph and $E_{d}, E_{s}$ the edges of $C_{G}$ that are forced by diamonds and splits. The graph $G$ admits a good partition, iff $C_{G}$ has a perfect matching $M$, with $\left(E_{d} \cup E_{s}\right) \subseteq M$.

Proof. The proof almost follows from Proposition 1 and the above considerations. Splitting connector vertices of degree 4 , however, may separate diamonds. We claim that this is no problem. Let $v$ be split into $v^{\prime}, v^{\prime \prime}$ as above. Any diamond containing $v$ has a cycle $z$ with bus vertices $b_{1}$ and $b_{3}$. Condition (P3) for this diamond requires that $b_{1}$ and $b_{3}$ belong to the same class of a good partition. This requirement, however, is already implied by condition (P2) for the original connector vertex $v$. That is, separated diamonds do not impose additional conditions on the matching.

Theorem 1. Let $G$ be a maximal plane bus graph. A good partition for $G$ can be computed in $O\left(n^{3 / 2}\right)$ time, if it exists.

Proof. By Proposition 2 it suffices to test the connector graph $C_{G}$ for a perfect matching $M$ that contains $\left(E_{d} \cup E_{s}\right)$. The extraction of the connector graph $C_{G}$ from $G$ requires linear time. The set $E_{s}$ can be computed while constructing $C_{G}$. To identify diamonds we consider the dual $D_{G}$ of the connector graph $C_{G}$. The vertices of $D_{G}$ are the bus vertices of $G$ and edges correspond to faces of $G^{\prime}$. Diamonds of $G^{\prime}$ corresponds to a double edge of $D_{G}$ the only exception is the diamond bounding the outer face. Double edges of $D_{G}$ can be found and sorted so that the set $E_{d}$ can be constructed in $O(n$ polylog $(n))$ time. To force $E_{d} \cup E_{s}$ we simply delete all vertices incident to 

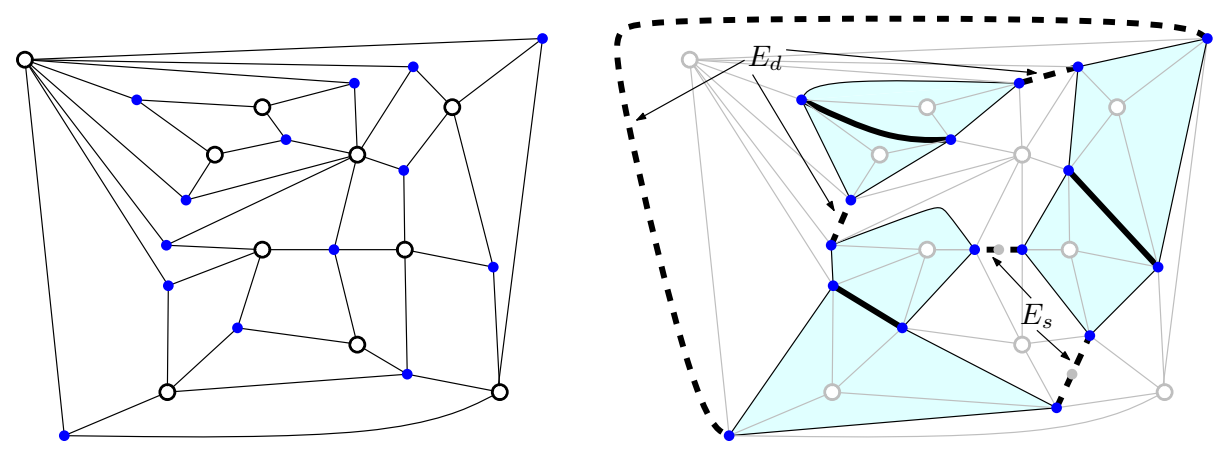

Fig. 3: A maximal plane bus graph and its connector graph with the modifications, where the dotted edges are forced and the fat edges complete the perfect matching.

these edges from the graph. If a vertex is incident to two edges from the set, then there is no matching. For constructing a maximum matching of a graph there exist several $O(\sqrt{n} m)$ algorithms. For planar graphs this yields the claimed $O\left(n^{3 / 2}\right)$ complexity. ${ }^{3}$

Given the perfect matching the corresponding good partition can again be computed in linear time.

\section{Non Maximal Plane Bus Graphs}

In this section we consider a plane bus graph $G$, that is not necessarily maximal. In a first preprocessing step we remove all bus vertices and connector vertices with degree 1 , as well as their incident edge. These objects can easily be integrated in a realization of the remaining graph.

In the following we describe how to extend $G$ to a maximal plane bus graph $G^{+}$ containing $G$ as induced subgraph such that $G^{+}$has a good partition iff $G$ has a good partition (Lemma 2). The graph $G^{+}$will be called the quadrangulation of $G$.

Let $f$ be a face with cardinality $2 k$ in $G$ and let $b_{1}, \ldots, b_{k}$ be the bus vertices of $f$ in clockwise order. To quadrangulate $f$ we first place a new bus vertex $b_{f}^{*}$ in the inside. The bus vertex $b_{f}^{*}$ is then connected to the boundary of $f$ by adding a triangular structure for every consecutive pair $b_{i}, b_{i+1}$ of bus vertices including the pair $b_{k}, b_{1}$. The triangular structure for $b_{i}, b_{i+1}$ consists of another new bus vertex $c_{i}$ and three connector vertices $v_{i}^{1}, v_{i}^{2}, v_{i}^{3}$ such that $N\left(v_{i}^{1}\right)=\left\{b_{i}, c_{i}, b_{i+1}\right\}, N\left(v_{i}^{2}\right)=\left\{b_{i+1}, c_{i}, b_{f}^{*}\right\}$, and $N\left(v_{i}^{1}\right)=\left\{b_{f}^{*}, c_{i}, b_{i}\right\}$. Figure 4 shows an example.

The graph $G^{+}$is obtained from $G$ by quadrangulation every face $f$ with cardinality $>4$ including, if necessary, the outer face. The following properties of the quadrangulation $G^{+}$of $G$ are obvious:

- $G^{+}$is planar and has $O(n)$ vertices.

- All diamonds of $G^{+}$are diamonds of $G .^{4}$

\footnotetext{
${ }^{3}$ In [13] a slightly faster randomized algorithm for planar graphs has been proposed.

${ }^{4}$ Note that the outer face of $G^{+}$has cardinality 4 if the outer face of $G$ has cardinality $>4$ this is an additional diamond of $G^{+}$. We ignore this diamond and the condition imposed by it on good partitions of $G^{+}$.
} 


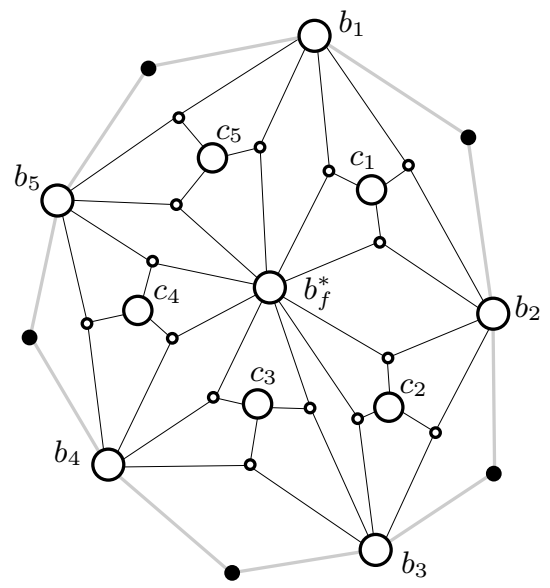

Fig. 4: New vertices and edges added to quadrangulate a face $f$ with cardinality 10 .

In addition we have the following important lemma:

Lemma 2. Let $G$ be a plane bus graph and $G^{+}$be its quadrangulation. Then $G$ has a good partition, iff $G^{+}$has a good partition.

Proof. The three defining properties (P1), (P2), and (P3) are stable under taking induced subgraphs. Hence, a good partition of $G^{+}$immediately yields a good partition of $G$.

Now assume that $G$ has a good partition. We aim for a partition of the bus vertices of $G^{+}$that extends the given partition of the bus vertices of $G$. Since all bus vertices of degree 4 and all diamonds of $G^{+}$already belong to $G$ we don't have to care of (P2), and (P3). The following rules define the labels for the new bus vertices such that (P1) is fulfilled for all new connector vertices:

- Label all central bus vertices $b_{f}^{*}$ with $V$.

- If $b_{i}$ and $b_{i+1}$ are both labeled $H$, then the label of $c_{i}$ is defined to be $V$. Otherwise the label $c_{i}$ is $H$.

It is straightforward to check that this yields a good partition of $G^{+}$.

If vertices have been added to the outer face $f^{*}$ in the quadrangulation process, then we can choose the outer face of $G^{+}$such that it contains $b_{f^{*}}^{*}$ and both bus vertices of the (new) outer face are labelled $V$. This change in the outer face does not affect the plane embedding of $G$. These considerations imply that when looking for a good partition of $G^{+}$we may disregard the condition implied by the diamond defined by the outer face if the outer face of $G$ had cardinality $>4$, c.f. footnote 4 .

Theorem 2. Let $G$ be a plane bus graph. A good partition for $G$ can be computed in $O\left(n^{3 / 2}\right)$ time, if it exists.

Proof. By Lemma 2 it suffices to test if the quadrangulation $G^{+}$of $G$ has a good partition. Hence we first compute $G^{+}$in linear time. Since $G^{+}$is a maximal plane bus graph we can use the algorithm from Theorem 1 to check whether $G^{+}$has a good partition. The running time is $O\left(n^{3 / 2}\right)$ and the algorithm returns a good partition if it exists. 


\section{Planar Realizations}

In the last two sections we analyzed the complexity of testing and computing a good partition. In this section we assume the existence of a good partition for a plane bus graph $G$ and give a polynomial time algorithm to construct a planar realization for $G$.

Theorem 3. Let $G$ be a plane bus graph admitting a good partition. Then $G$ has a planar realization on an $O(n) \times O(n)$ grid. If the good partition is given the realization can be computed in $O(n)$ time.

Let $G$ be a plane bus graph admitting a good partition. Again we start with some simplifications. First we recursively remove all connector and bus vertices of degree 1 and all connector vertices of degree 2 .

Let $G^{+}$be the quadrangulation of $G$. The assumption about the existence of a good partition of $G$ together with Lemma 2 imply that $G^{+}$has a good partition which can by Theorem 1 be computed efficiently.

Given the good partition of $G^{+}$we split all connector vertices of degree 4 into two connector vertices of degree 3 . For simplicity we continue denoting the resulting graph $G^{+}$.

The reduced bus graph $R^{+}=\left(B^{+}, E_{R}\right)$ of $G^{+}$is the graph on the bus vertices of $G^{+}$with edges $\left(b, b^{\prime}\right)$, iff $b, b^{\prime}$ are incident to a common face and have different labels. Diamonds with different labeled bus vertices are the only substructure that would create double edges in $R^{+}$but diamonds have identically labeled bus vertices in a good partition. Hence, there are no double edges in $R^{+}$. From the three faces incident to a connector vertex exactly two contribute an edge to $R^{+}$. It follows that $R^{+}$is a quadrangulation, i.e., all faces have cardinality 4 . Another approach to derive this is by observing that the edges of the matching $M$ of Proposition 2 are in bijection with the faces of $R^{+}$.

Let $Q$ be a quadrangulation, we call the color classes of the bipartition white and black and name the two black vertices on the outer face $s$ and $t$. A separating decomposition of $Q$ is an orientation and coloring of the edges of $Q$ with colors red and blue such that:

- All edges incident to $s$ are ingoing red and all edges incident to $t$ are ingoing blue.

- Every vertex $v \neq s, t$ is incident to a non-empty interval of red edges and a nonempty interval of blue edges. If $v$ is white, then, in clockwise order, the first edge in the interval of a color is outgoing and all the other edges of the interval are incoming. If $v$ is black, the outgoing edge is the last one in its color in clockwise order (see Figure 5).
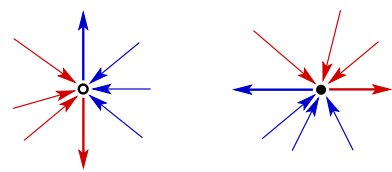

Fig. 5: Edge orientations and colors at white and black vertices. 
Separating decompositions have been studied in [2], [6], and [5]. In particular it is known that every plane quadrangulation admits a separating decomposition. To us separating decompositions are of interest because of their connection with segment contact representations of the underlying quadrangulation. A proof of the following lemma can be found in [4].

Lemma 3. A separation decomposition of $Q$ can be used to construct a segment contact representation of $Q$ with vertical and horizontal segments, such that edges $v \rightarrow w$ of the separating decomposition correspond to a contact of the segments $S_{v}$ and $S_{w}$ where an endpoint of $S_{v}$ is in the interior of $S_{w}$.

An illustration for the lemma is given in Figure 6.
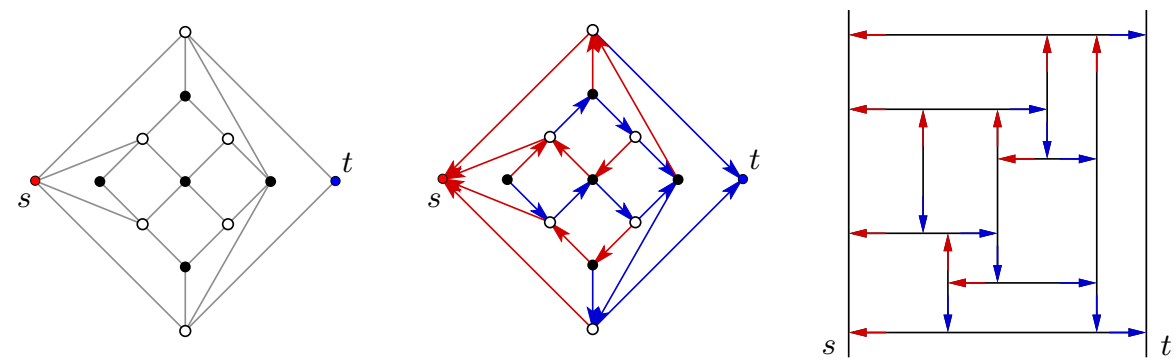

Fig. 6: A quadrangulation $Q$, a separating decomposition of $Q$ and a corresponding segment contact representation of $Q$.

Identify the two classes $V$ and $H$ of the bipartition of the reduced bus graph $R^{+}$ with black and white. Construct a separating decomposition of $R^{+}$and a corresponding segment contact representation. Later the following observation will be important:

$(\star)$ The rectangles in the segment contact representation correspond bijectively to the faces of $R^{+}$. Moreover, vertex $b$ is incident to face $f$ in $R^{+}$iff segment $S_{b}$ contributes a side of the rectangle $R_{f}$ corresponding to $f$.

From the segment contact representation of $R^{+}$we obtain a representation of the bus graph $G^{+}$in two steps. First clip the endpoints of all segments of the representation so that a disjoint collection of segments remains. These segments serve as the bus segments for the representation of the bus graph $G^{+}$. It remains to insert the connector vertices and the edges of $G^{+}$into the picture. To this end recall that each connector vertex belongs to a unique face of $R^{+}$and each face of $R^{+}$contains exactly two connector vertices. The two connector vertices contained in a face $f$ can easily be accommodated in the rectangle $R_{f}$, because of ( $\star$ ). Figure 7 shows the picture.

At this point we have a representation of the plane bus graph $G^{+}$. It remains to transform this into a representation of the original input graph $G$. These are the steps that have to be done:

- Merge pairs of connector vertices that have been created by splitting a connector vertex of degree 4 . 

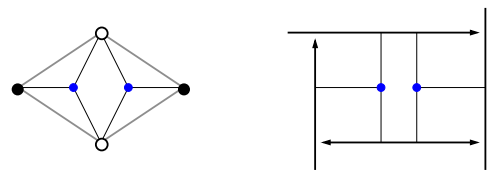

Fig. 7: A face $f$ of $R^{+}$with its two connector vertices and the placement of the two vertices in $R_{f}$.

- Delete all bus and connector vertices from the representation that have been introduced to make the bus graph maximal.

- Insert all connector and bus vertices of degree 1 and all connector vertices of degree 2 that had been deleted in the reverse order of the deletion.

This yields a representation of the input graph $G$.

To complete the proof of Theorem 3 it remains to argue about the complexity. Let $G=(V \cup B, E)$ be the input bus graph with $n=|V|+|B|$. Simple estimates on the basis of Euler's formula show that in going from $G$ to $G^{+}$at most $14|B|$ new vertices have been introduced, hence, $G^{+}$has $n^{+} \in O(n)$ vertices. The reduced bus graph $R^{+}$ can be computed from the plane $G^{+}$in $O\left(n^{+}\right)$. A separating decomposition of $R^{+}$can also be computed in linear time, details can be found in the $\mathrm{PhD}$ thesis of É. Fusy [7]. The segment contact representation of $R^{+}$associated to the separation decomposition is computable in linear time with standard techniques, c.f. [3] or [4]. The number of grid lines needed for the segment contact representation of $R^{+}$is bounded by the number of bus vertices of $R^{+}$, i.e., the size of the grid is $O(n) \times O(n)$. The clipping of endpoints increases the number of grid lines by a factor of 3 and the insertion of connector vertices may require an additional grid line for each vertex. The same holds for the reinsertion of vertices of degree 1 and 2 . All these steps can be done in linear time and keep the size of the grid in $O(n) \times O(n)$.

\section{Conclusion and Future Work}

We have considered the class of plane bus graphs, that admit a planar realization and have characterized this class by the existence of a good partition of bus vertices. To test for the existence of a good partition we gave an $O\left(n^{3 / 2}\right)$ algorithm based on planar matching. Given a good partition the representation can be computed in linear time.

It would be interesting to extend the approach from plane to planar bus graphs. The problem here is that the connector graphs of different plane embeddings of a planar graph differ.

It is also open to characterize the class of graphs that admit realizations, where connections are allowed to cross apart from the knowledge, that the decision is NPcomplete. Another generalization would be to allow connections to cross bus segments or bus segments to cross each other. 


\section{References}

1. Ada, A., Coggan, M., Marco, P.D., Doyon, A., Flookes, L., Heilala, S., Kim, E., Wing, J.L.O., Préville-Ratelle, L.F., Whitesides, S., Yu, N.: On bus graph realizability. CCCG abs/cs/0609127, 229-232 (2007)

2. De Fraysseix, H., De Mendez, P.O.: On topological aspects of orientations. Discrete Mathematics 229(1-3), 57-72 (2001)

3. Di Battista, G., Eades, P., Tamassia, R., Tollis, I.G.: Graph Drawing: Algorithms for the Visualization of Graphs. Prentice-Hall (1999)

4. Felsner, S.: Rectangle and square representations of planar graphs. In: Pach, J. (ed.) Thirty Essays in Geometric Graph Theory, Algorithms and Combinatorics, vol. 29. Springer Verlag (2012)

5. Felsner, S., Fusy, É., Noy, M., Orden, D.: Bijections for Baxter families and related objects. Journal of Comb. Theory A 18, 993-1020 (2011)

6. Felsner, S., Huemer, C., Kappes, S., Orden, D.: Binary labelings for plane quadrangulations and their relatives. Discrete Mathematics \& Theoretical Computer Science 12(3), 115-138 (2010)

7. Fusy, E.: Combinatoire des cartes planaires et applications algorithmiques. Ph.D. thesis, LIX Ecole Polytechnique (2007)

8. Hanan, M.: On Steiner's problem with rectilinear distance. SIAM J. Appl. Math (14), 255265 (1966)

9. He, X.: On finding the rectangular duals of planar triangular graphs. SIAM J. Comput 22, 1218-1226 (1993)

10. Hwang, F.W., Richards, D.S., Winter, P.: The Steiner tree problem. Annals of Discrete Mathematics (53) (1992)

11. Kaufmann, M., Wagner, D. (eds.): Drawing Graphs, Methods and Models, Lecture Notes in Computer Science, vol. 2025. Springer (2001)

12. Lengauer, T.: VLSI theory. In: Handbook of Theoretical Computer Science, Volume A: Algorithms and Complexity (A), pp. 835-868 (1990)

13. Mucha, M., Sankowski, P.: Maximum matchings in planar graphs via gaussian elimination. Algorithmica 45(1), 3-20 (2006)

14. Nishizeki, T., Rahman, M.S.: Planar Graph Drawing. World Scientific (2004)

15. Rosenstiehl, P., Tarjan, R.E.: Rectilinear planar layouts and bipolar orientations of planar graphs. Discrete \& Computational Geometry 1, 343-353 (1986)

16. Tamassia, R., Tollis, I.G.: A unified approach a visibility representation of planar graphs. Discrete \& Computational Geometry 1, 321-341 (1986)

17. Thompson, C.D.: A Complexity Theory for VLSI. Ph.D. thesis, Carnegie-Mellon University (1980)

18. Zhou, X., Nishizeki, T.: Algorithm for the cost edge-coloring of trees. J. Comb. Optim. 8(1), 97-108 (2004) 